\title{
Stage IV choriocarcinoma. Case report and review of
}

literature.

$\begin{gathered}\text { A. Veiga-Fernández', C. García-Díaz', J.Á. Arranz }{ }^{2}, \text { E.M. Mendizábal-Vicente' } \\ \text { P. Rincón-Olbes' }\end{gathered}$, S. Lizarraga-Bonelli1
1Obstetrics and Gynaecology, ${ }^{2}$ Medical Oncology.
Gregorio Marañón University General Hospital, Madrid, Spain

\section{Introduction/Background:}

Choriocarcinoma is the most aggressive histologic type of gestational trophoblastic neoplasia (GTN). It consists of invasive, highly vascular and anaplastic trophoblastic tissue made up of cytotrophoblasts and syncytiotrophoblasts without villi, and it is characterized by early vascular invasion and widespread metastases.

\section{Methods:}

We present a clinical case and a review of literature.

\section{Results:}

A 33-years-old woman with 2 previous caesarean deliveries, last one in July 2018, was referred to our hospital in December 2018 after a seizure secondary to a brain tumour. Human chorionic gonadotropin (b-hCG) was raised with a normal gynaecological ultrasound except for a suspicious sonographic image of ectopic pregnancy. She was treated with one dose of Methotrexate, without decrease of b-hCG levels. Finally, the patient underwent brain surgery and the tumour was completely resected (Images 1 and 2). After that, b-hCG decreased quickly.

Histological study showed a choriocarcinoma. No primary tumour was found in complementary imaging tests.

Choriocarcinoma occurs in about 1 in 20,000 to 40,000 pregnancies in the United States and three to nine per 40,000 pregnancies in Southeast Asia and Japan. About $50 \%$ of all choriocarcinomas arise from a complete molar gestation, $25 \%$ following a normal pregnancy, and $25 \%$ after a spontaneous miscarriage or ectopic pregnancy.

Diagnosis is often delayed following a nonmolar pregnancy and thus, metastases are more common than after a molar pregnancy.

Clinical presentation of choriocarcinoma depends on the extent of disease and location of metastases. The most frequent sites of gestational trophoblastic neoplasias metastases are: lung $(80 \%)$, vagina $(30 \%)$, central nervous system (10\%) and liver $(10 \%)$.

\section{Conclusion:}

Choriocarcinoma is a rare disease. Patients should be managed by a gynaecologist oncologist and/or medical oncologist experienced with GTN.

Treatment includes chemotherapy: etoposide, methotrexate, actinomycin $D$, cyclophosphamide and vincristine, combined with uterine surgery.

According to the International Federation of Gynecology and Obstetrics, the majority of cases of choriocarcinoma are chemotherapy sensitive, with a survival rate of nearly $80-100 \%$.

\section{Disclosures: No conflicts of interest to disclose.}
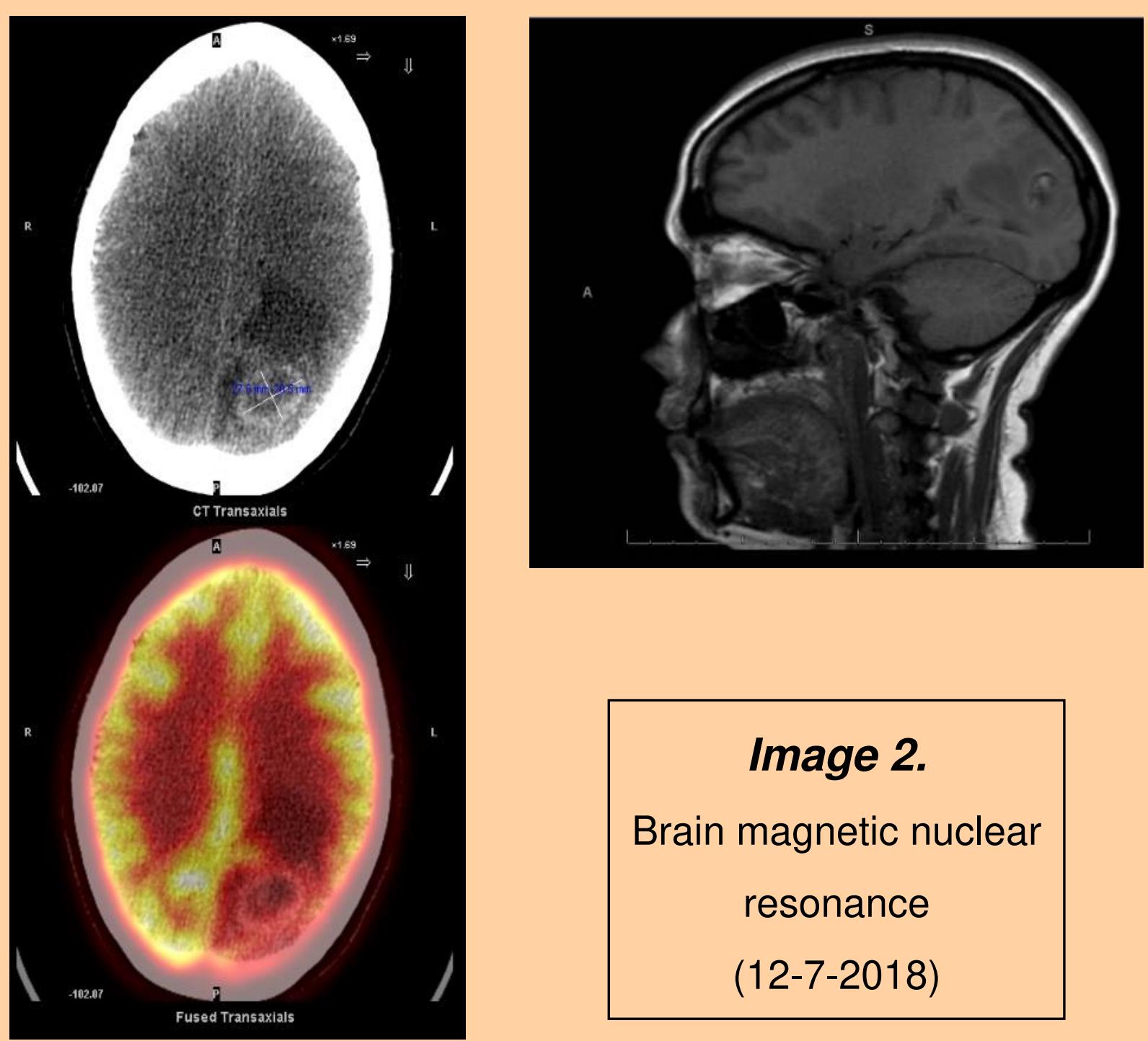

Image 2.

Brain magnetic nuclear resonance (12-7-2018)

\section{Image 1.}

PET fused transaxials (12-27-2018) 